\title{
HINDGUT CHANGES PRECEDING PUPATION AND RELATED COCOON STRUCTURE IN CHRYSOPERLA COMANCHE BANKS (NEUROPTERA, CHRYSOPIDAE).
}

\author{
By Craig LaMunyon* \\ Department of Biology, California State University \\ Fullerton, CA 92635
}

\section{INTRODUCTION}

A blockage occurs between the midgut and hindgut of larval neuropterans in the suborder Planipennia (New 1986). Thus, the feces are stored in the midgut during larval life, enabling the hindgut to perform other functions. A compound stored in the hindgut of some larval chrysopid species is extruded from the abdominal tip and used as an adhesive during locomotion (Spiegler 1962) and for defense when the larvae are attacked (LaMunyon and Adams 1987).

This adhesive/defensive substance is produced by the malpighian tubules of chrysopid larvae. However, as pupation nears, the malpighian tubules produce silk precursors (Spiegler 1962), and undergo a dramatic histological change in the ca. 2 day transformation from mature larva to prepupa (the cocoon building stage) (McDunnough 1909, Spiegler 1962, Wigglesworth 1972). The silk precursors flow from the tubules into the hindgut and are extruded from the anus during cocoon construction.

The composition of chrysopid cocoons is not known. Larval silk does not have the solubility properties of protein (Lucas and Rudall 1968), and Rudall and Kenchington (1971) suggested it might be "a kind of nylon with condensation of dicarboxylic and diamino hydrocarbons." These authors also found that cocoons are composed of an outer fibrous silk, and an inner wall consisting of compact layers. Infrared spectroscopy suggested that the inner wall is composed of a polymerized molecule containing some alpha helical domains. They placed the cocoons in the category of the cuticulin silks, which are produced by epithelial cells.

*Present address: Section of Neurobiology and Behavior, Cornell University, Ithaca, NY 14853-2702

Manuscript received by the editor September 9, 1988. 
While performing a histological examination of the abdomen of C. comanche larvae, I found that the hindgut epithelium changes drastically prior to pupation. The work described here is a light microscopic examination of larval and prepupal C. comanche hindgut epithelium, and an electron microscopic investigation of cocoon structure.

\section{Materials AND Methods}

Laboratory colonies of $C$. comanche were raised from adults collected in Orange Co., Ca. Rearing procedures followed those described earlier (LaMunyon and Adams 1987).

Mid-third instar larvae, prepupae spinning silk, and prepupae that had just spun cocoons, were prepared for light microscopy in
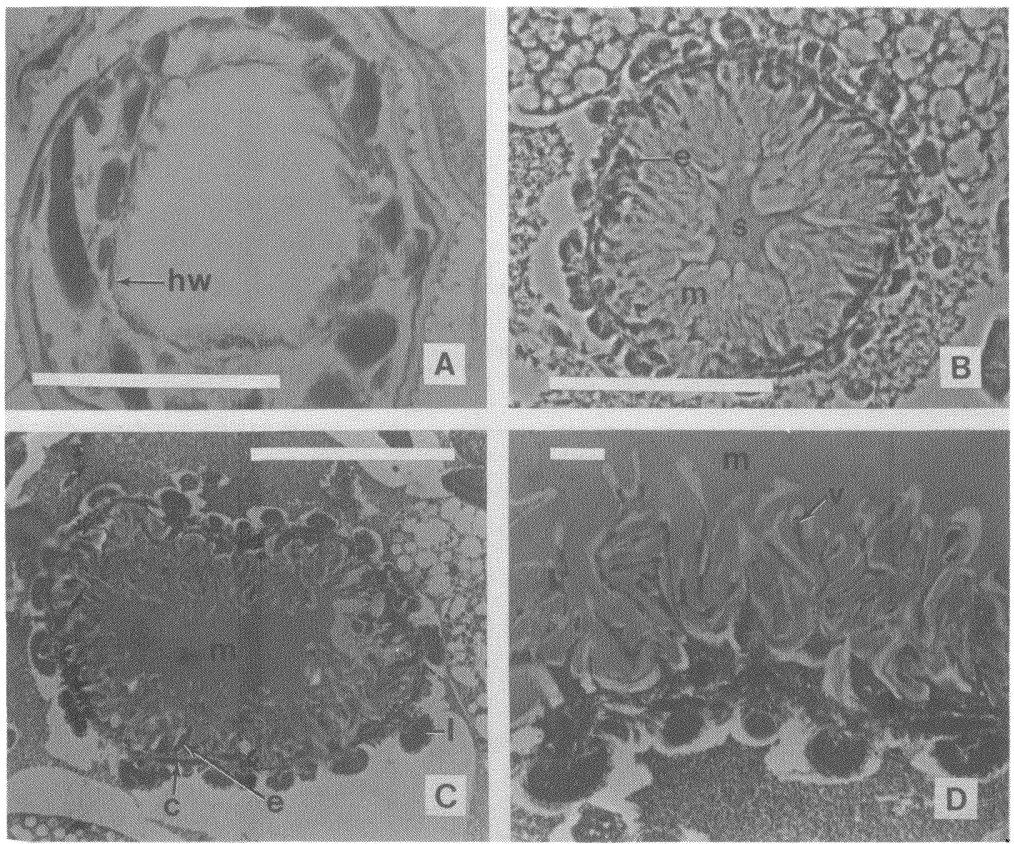

Figure 1. Light micrographs of cross sections through the abdomen of larval and prepupal C. comanche. (A) Mid third-instar larva $(\mathrm{Bar}=100 \mu)$. (B) Prepupa spinning silk (phase contrast; Bar $=100 \mu)$. $(\mathrm{C})$ Prepupa after spinning silk $(\mathrm{Bar}=100 \mu)$. (D) Close-up of $(\mathrm{C})(\mathrm{Bar}=10 \mu)$. c, circular muscle; e, epithelium; hw, hindgut wall; l, longitudinal muscle; $m$, epithelial associated material; s, silk precursors; v, epithelial villi. 
two ways. Some were fixed in formalin, dehydrated in ethanol, embedded in paraffin, sectioned at 8 microns, then mounted and stained with hematoxylin and eosin. Others were fixed in $4 \%$ glutaraldehyde in $0.01 \mathrm{M}$ phosphate buffer $\left(\mathrm{pH} 7.2\right.$ at $\left.5^{\circ} \mathrm{C}\right)$, post fixed in $2 \%$ osmium tetroxide in $0.01 \mathrm{M}$ phosphate buffer $(\mathrm{pH} 7.2$ at room temperature), dehydrated in ethanol, embedded in Epon-Araldite 502 , sectioned at 1 micron, and mounted and stained with toluidine blue. In all, 12 larvae and 6 prepupae were sectioned and examined.

Empty cocoons were also examined microscopically. Some were embedded in paraffin, sectioned at $\mathbf{4 0}$ microns, then mounted and stained with eosin. Others were fractured after submersion in liquid nitrogen, mounted on stubs, sputter-coated with gold, and viewed with a scanning electron microscope.

\section{Results}

In mature third-instar larvae, the defensive substance is stored in the hindgut, which has a very thin epithelium (Fig. 1A). In prepupae spinning silk, the epithelium is greatly enlarged and villous, extending into the lumen (Fig. 1B). Two substances are visible in this section: an innermost eosin-staining material continuous with malpighian tubule-produced silk precursors, and an outer, non-staining substance associated with the villous epithelium. In prepupae fixed after spinning the outer layer of cocoon fibers, the epithelium is still unusually enlarged, and long, finger-like extensions into the lumen are visible (Fig. 1C \& 1D). At this stage, only the epitheliumassociated substance remains.

In the paraffin cocoon sections, there were two different materials present: the outermost silk which stained with eosin, and the nonstaining substance making up the solid inner wall (micrographs not included). These two cocoon layers appear discrete in the electron micrographs. The inner wall is relatively thick, is surrounded by the fibrous silk, and is the major component of the cocoon (Fig. $2 \mathrm{~A}, \mathrm{~B}$, C). Figure $2 \mathrm{C}$ also shows a pore through the inner wall; all pores observed were composed of two holes. The homogeneity of the inner wall is evident from Fig. 2D.

The abdominal musculature changes during transition from mature, third instar larvae to prepupae. Large, intersegmental muscles can be seen traversing the section of a younger larva (Fig. 1A). The defensive substance, being less viscous than cocoon precursors 

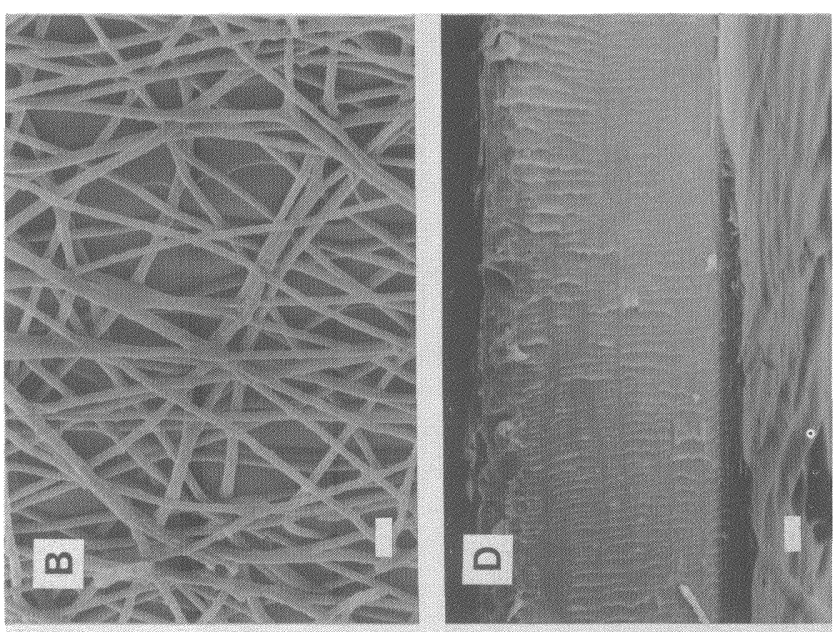

害范

11

忥导

造

氖

호

응

II

壱

ङ

ํํㄹ

迟

气ัँ

है

$8 \%$

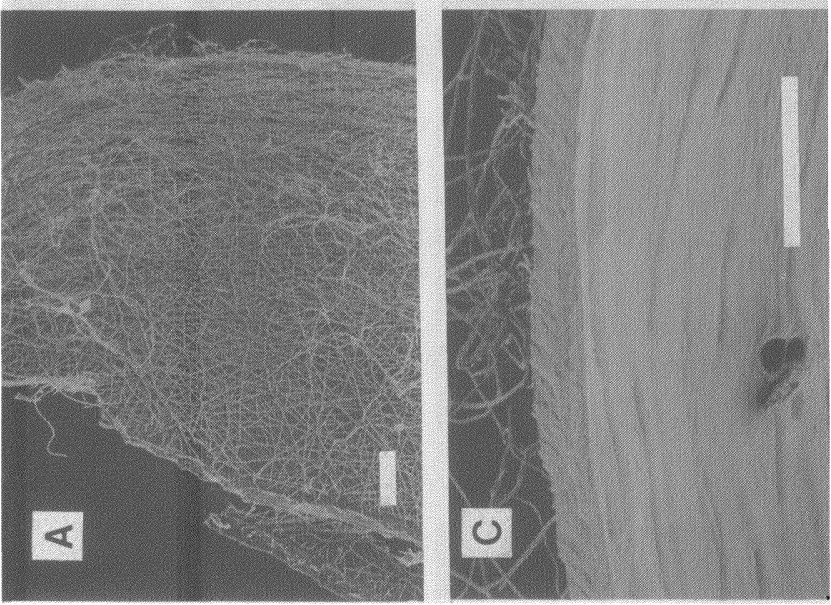

ن

ग

롫 몷

跑

엉

논

उั 点 ต 을 온 총. 응 동 0

包

즐 论 극용 ㄱ 鸟 ญ 偪它 
(personal observation), is probably expelled by compression of the entire abdomen. In prepupae, the circular and longitudinal hindgut muscles are enlarged and much more evident (Fig. 1B, 1C), and probably expel the more viscous cocoon precursors by compressing only the hindgut.

\section{Discussion}

The hindgut epithelium changes quickly from a very thin layer to a villous tissue that is probably secretory. This inference is supported by two facts. First, the malpighian tubules are the only organs opening proximally into the hindgut; these produce precursors of the fibrous silk, which stain differently than the epitheliumassociated substance. Thus, the only tissue apparently responsible for the production of this latter substance would be the villous hindgut epithelium. Second, the appearance of this material coincides with the transformation of the epithelium (presumably to a secretory villous phase). The villous extensions are probably involved in secretion, and could be detaching from the cells and breaking open into the lumen.

Insect hindgut epithelia normally secrete a thin layer of cuticle prior to molting, and the epithelium-associated material may be similar to some cuticle component. This finding supports the placement of chrysopid silk (pertaining to the epithelial substance) with the epithelial-produced cuticulin silks (Rudall and Kenchington 1971). Other insect hindguts with a secretory function are known; in some tephritid flies a pheromone is secreted by a derived hindgut epithelium (Little and Cunningham 1987).

It appears that the fibrous silk of the cocoon is produced by the malpighian tubules; the inner wall may be formed from the epithelium-associated substance. This hypothesis is supported by the temporal changes in the hindgut. The eosinophylic, malpighian tubule-produced silk precursors are found in the lumen during spinning of the fibrous silk. Once the silk layer appears complete, only the epithelium-associated material remains in the hindgut. This stage may coincide with the time when the inner wall is laid down. The gross physical nature of the cocoons also supports this hypothesis. After completion of the fibrous silk layer, the cocoons are very soft; ca. one day later, they become hard, probably as the inner wall is formed. 
The fibrous silk probably provides a substrate for the formation of the solid inner wall which is the major cocoon component and has periodic perforations. Aside from mechanical protection, the functions of this wall may include resistance to infection, parasitoid oviposition, and desiccation. It is not known if the inner wall of more mesic species is also the major cocoon component, but $C$. comanche inhabits an arid environment (southern CA), in which desiccation protection can be expected to be vital for the two week metamorphic period. On the other hand, there is need for gas exchange: hence, the pores.

\section{ACKNOWLEDGMENTS}

I would like to thank S. Karl for his help with plastic embedding and electron microscopy, P. Adams, C. Lambert, and T. Eisner for their help with light microscopy, and M. Eisner for her help with electron microscopy. This manuscript was improved by the reviews of I. Baldwin, S. Smedley, T. Eisner and P. Adams. This work was supported by grants from the Department of Biology, California State University, Fullerton.

\section{SUMMARY}

The hindgut epithelium in prepupal $C$. comanche becomes unusually villous and may be secreting a substance found in the hindgut lumen. This substance is probably used to form the solid inner cocoon wall; both the inner cocoon wall and the hindgut substance have similar staining properties. Malpighian tubule-produced silk precursors stain similarly to the outer, fibrous cocoon silk. Hence, cocoons appear to be composed of two different substances secreted by different tissues. Cocoon structure is also described.

\section{Literature Cited}

LaMunyon, C. W., and P. A. Adams.

1987. Use and effect of an anal defensive secretion in larval Chrysopidae (Neuroptera). Ann. Entomol. Soc. Am. 80(6): 804-808.

Little, H. F., AND R. T. CUNningham.

1987. Sexual dimorphism and presumed pheromone gland in the rectum of Dacus latifrons (Diptera: Tephritidae). Ann. Entomol. Soc. Am. 80(6): 765-767. 
LuCAs, F., AND K. M. Rudall.

1968. Extracellular fibrous proteins: the silks. In: Comprehensive Biochemistry, vol. 26B, Ch. 7. Eds. M. Florkin and E. H. Stotz. Elsevier, Amsterdam. pp. 475-558.

McDunnough, $\mathbf{J}$.

1909. Über den Bau des Darms und seiner Anhange von Chrysopa perla L. Arch. Naturgesch. 75: 313-360.

New, T. R.

1986. A review of the biology of Neuroptera Planipennia. Neuroptera International, Suppl. Ser. 1.

RudDall, K. M., AND W. Kenchington.

1971. Arthropod silks: the problem of fibrous proteins in animal tissues. Annu. Rev. Entomol. 16: 73-96.

SPIEGLER, P. E.

1962. The origin and nature of the adhesive substance in larvae of the genus Chrysopa (Neuroptera: Chrysopidae). Ann. Entomol. Soc. Am. 55: 69-77.

WIGGLESWORTH, V. B.

1972. The principles of insect physiology. Chapman and Hall, London. 827 pp. 

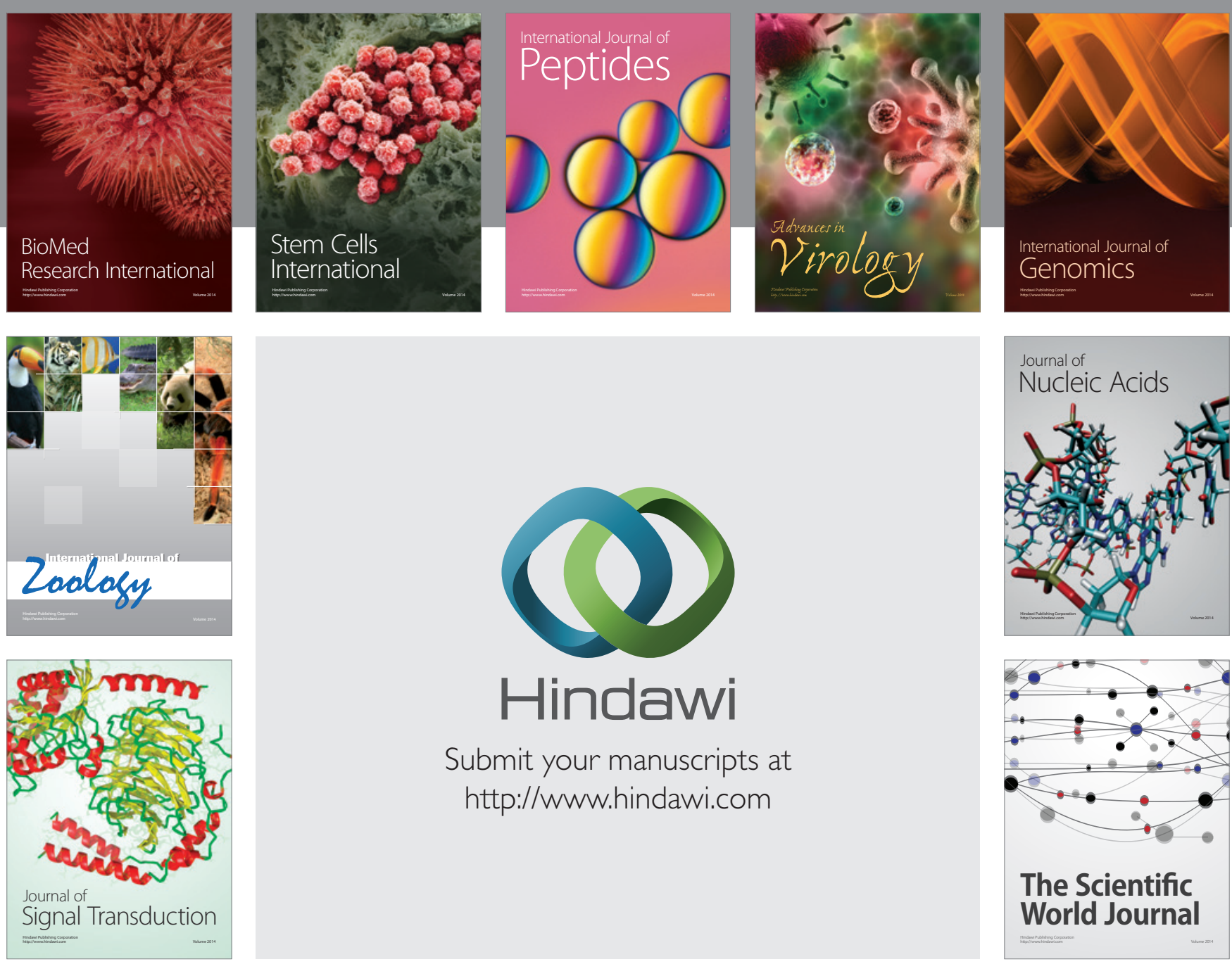

Submit your manuscripts at

http://www.hindawi.com
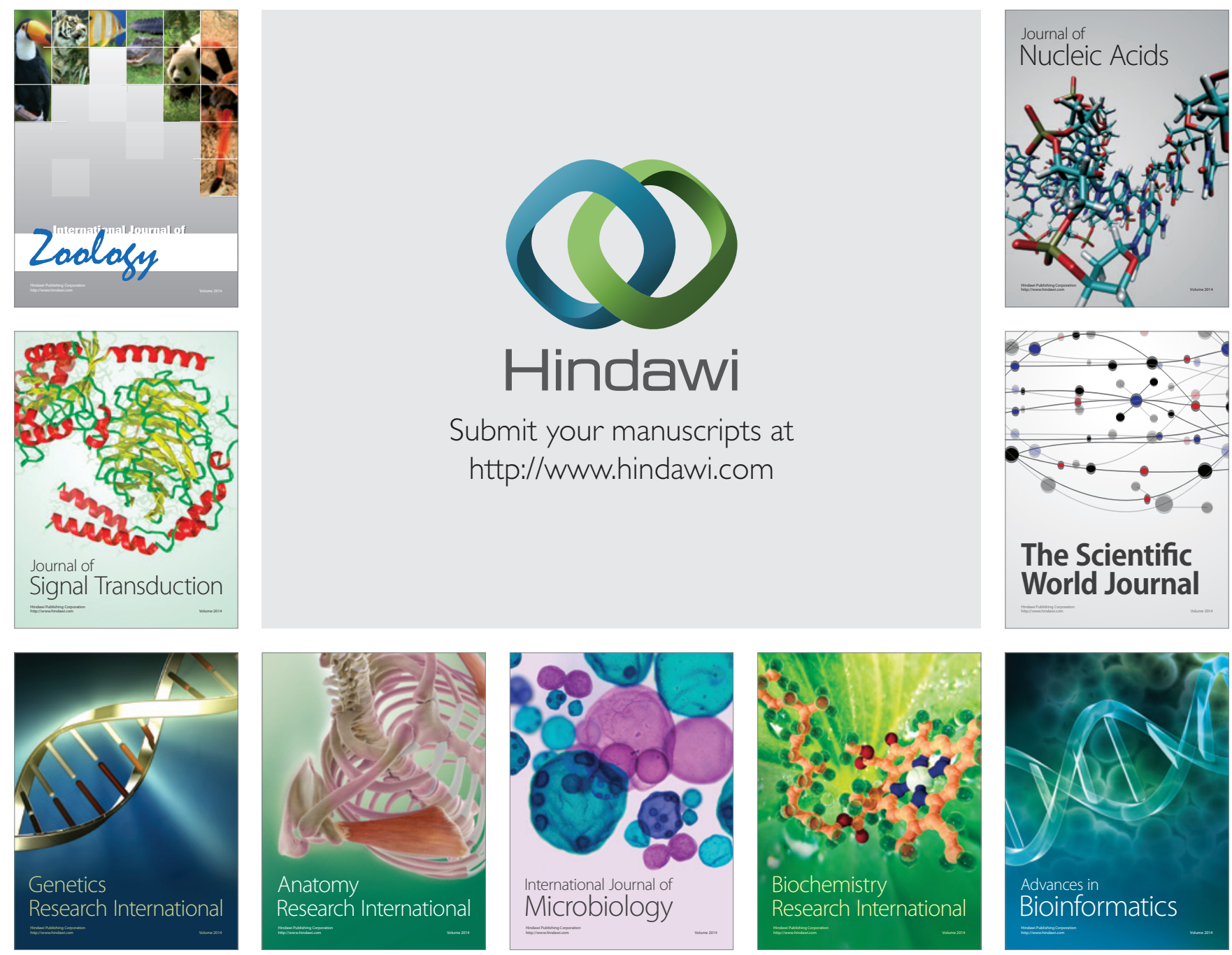

The Scientific World Journal
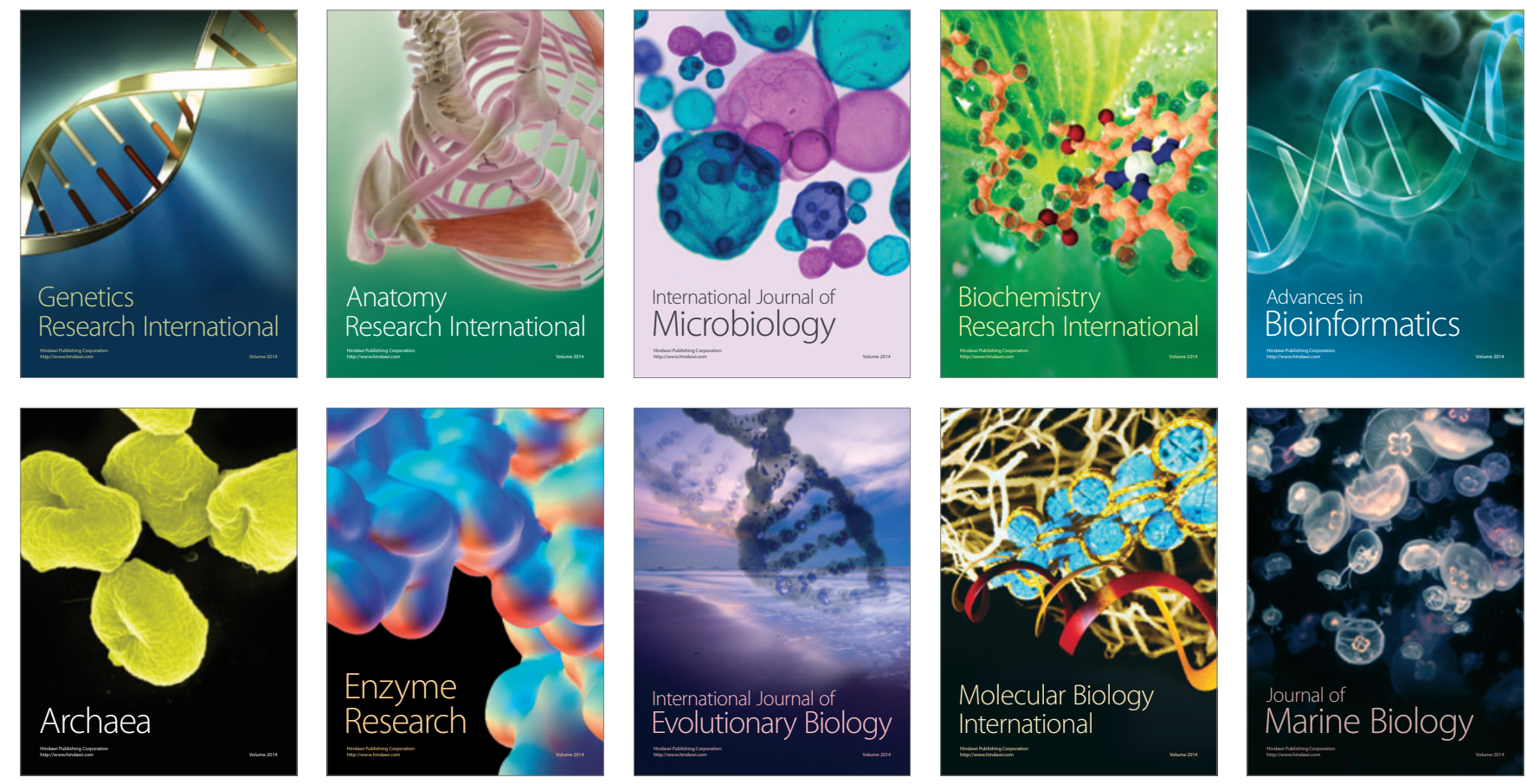